\title{
THE GENERALISED COUPON COLLECTOR PROBLEM
}

\author{
PETER NEAL, ${ }^{*}$ University of Manchester
}

\begin{abstract}
Coupons are collected one at a time from a population containing $n$ distinct types of coupon. The process is repeated until all $n$ coupons have been collected and the total number of draws, $Y$, from the population is recorded. It is assumed that the draws from the population are independent and identically distributed (draws with replacement) according to a probability distribution $X$ with the probability that a type- $i$ coupon is drawn being $\mathrm{P}(X=i)$. The special case where each type of coupon is equally likely to be drawn from the population is the classic coupon collector problem. We consider the asymptotic distribution $Y$ (appropriately normalized) as the number of coupons $n \rightarrow \infty$ under general assumptions upon the asymptotic distribution of $X$. The results are proved by studying the total number of coupons, $W(t)$, not collected in $t$ draws from the population and noting that $\mathrm{P}(Y \leq t)=\mathrm{P}(W(t)=0)$. Two normalizations of $Y$ are considered, the choice of normalization depending upon whether or not a suitable Poisson limit exists for $W(t)$. Finally, extensions to the $K$-coupon collector problem and the birthday problem are given.
\end{abstract}

Keywords: Coupon collector problem; Poisson convergence; birthday problem

2000 Mathematics Subject Classification: Primary 60F05

Secondary $60 \mathrm{G} 70$

\section{Introduction}

The classic coupon collector problem has a long history; see, for example, [3]. The classic problem is as follows. A collector wishes to collect a complete set of $n$ distinct coupons, labelled 1 through to $n$. The coupons are hidden inside breakfast cereal boxes and within each cereal box there is one coupon which is equally likely to be any of the $n$ distinct coupons. The collector purchases one box of breakfast cereal at a time, collecting the coupons, stopping when the collector has completed the set of $n$ distinct coupons. The total number of cereal boxes, $Y_{n}$, which the collector needs to purchase is the quantity of interest. Elementary calculations show that

$$
\mathrm{E}\left[Y_{n}\right]=n \sum_{i=1}^{n} \frac{1}{i} \approx n \log n .
$$

Furthermore, if $Z$ is a standard Gumbel distribution with $\mathrm{P}(Z \leq z)=\exp \left(-\mathrm{e}^{-z}\right)(z \in \mathbb{R})$ then

$$
\frac{1}{n}\left(Y_{n}-n \log n\right) \stackrel{\mathrm{D}}{\rightarrow} Z \quad \text { as } n \rightarrow \infty,
$$

where ' $\stackrel{\mathrm{D}}{\rightarrow}$ ' denotes convergence in distribution; see, for example, [4].

Received 30 April 2008; revision received 27 May 2008.

* Postal address: School of Mathematics, University of Manchester, Alan Turing Building, Oxford Road, Manchester M13 9PL, UK. Email address: p.neal-2@manchester.ac.uk 
The generalised coupon collector problem assumes that, whilst the cereal boxes are independent and identically distributed, the probability that a box contains coupon $i$ is $p_{i}$. No assumption is placed upon the $\left\{p_{i}\right\}$ s except that $p_{i}>0(i=1,2, \ldots, n)$. We allow for the possibility that some boxes may not contain a coupon by only assuming that $\sum_{i=1}^{n} p_{i} \leq 1$. The random coupon collector problem [4], [5] is an alternative departure from the classic problem. The proofs in [4] rely upon a Poisson embedding argument and although our proofs are different we shall also exploit a Poisson approximation approach.

The paper is structured as follows. In Section 2 the main result, Theorem 2.1, is presented and proved. An alternative result is given in Theorem 2.2 which is applicable when the Poisson arguments of Theorem 2.1 fail. A number of examples are considered in Section 3. Finally, in Section 4 extensions of Section 2 are discussed. These include the $K$-coupon collector problem, the total number of draws from the population that are required to have $K$ coupons of each type, and the $K$-birthday problem, the total number of draws from the population that are required to have $K$ coupons of any (unspecified) type.

\section{Coupon collecting problem}

For the asymptotic results of this paper, we consider a sequence of coupon collections $\left\{\mathcal{C}_{n}\right\}$, where the number of coupons to be collected, $n$, tends to $\infty$. For $n \geq 1, \mathcal{C}_{n}$ requires the collection of $n$ coupons, labelled 1 through to $n$. Coupons are collected as follows. Let $X_{1}^{n}, X_{2}^{n}, \ldots$ be independent and identically distributed according to $X^{n}$, where

$$
\mathrm{P}\left(X^{n}=i\right)= \begin{cases}p_{n i}, & i=1,2, \ldots, n, \\ 0, & \text { otherwise }\end{cases}
$$

where $\sum_{i=1}^{n} p_{n i} \leq 1$ and $\min _{1 \leq i \leq n} p_{n i}>0$. Then $X_{k}^{n}$ is the $k$ th coupon drawn from the population (of coupons) and the process is continued until all $n$ coupons have been collected. Let $Y_{n}$ denote the total number of coupons which need to be collected to obtain the full set of coupons in $\mathcal{C}_{n}$.

Before stating the main result, we introduce some useful notation. For $n \geq 1, i=$ $1,2, \ldots, n$, and $t=1,2, \ldots$, let $\chi_{i}^{n}(t)=1$ if coupon $i$ has not been collected in the first $t$ coupons drawn from the population and $\chi_{i}^{n}(t)=0$ otherwise. Let $W_{n}(t)=\sum_{i=1}^{n} \chi_{i}^{n}(t)$, the total number of distinct coupons which still need to be collected after $t$ coupon draws. Thus, for $t \geq 1, Y_{n} \leq t$ if and only if $W_{n}(t)=0$.

Theorem 2.1. Suppose that there exist sequences $\left\{b_{n}\right\}$ and $\left\{k_{n}\right\}$ such that $k_{n} / b_{n} \rightarrow 0$ as $n \rightarrow \infty$ and that, for $y \in \mathbb{R}$,

$$
\sum_{i=1}^{n} \exp \left(-p_{n i}\left\{b_{n}+y k_{n}\right\}\right) \rightarrow g(y) \quad \text { as } n \rightarrow \infty
$$

for a nonincreasing function $g(\cdot)$ with $g(y) \rightarrow \infty$ as $y \rightarrow-\infty$ and $g(y) \rightarrow 0$ as $y \rightarrow \infty$. Then, if $\tilde{Y}_{n}=\left(Y_{n}-b_{n}\right) / k_{n}$,

$$
\tilde{Y}_{n} \stackrel{\mathrm{D}}{\rightarrow} Y \quad \text { as } n \rightarrow \infty,
$$

where $Y$ has cumulative distribution function

$$
\mathrm{P}(Y \leq y)=\mathrm{e}^{-g(y)}, \quad y \in \mathbb{R} .
$$


The key restriction in Theorem 2.1 is that (2.1) implies that $\min _{1 \leq i \leq n} p_{n i} b_{n} \rightarrow \infty$ as $n \rightarrow \infty$. This condition is needed for the Poisson limit (2.2), below, since it implies that $\max _{1 \leq i \leq n} \mathrm{E}\left[\chi_{i}^{n}\left(\left[b_{n}+y k_{n}\right]\right)\right] \rightarrow 0$ as $n \rightarrow \infty$. In Theorem 2.2, below, we explore the case where $\min _{1 \leq i \leq n} p_{n i} b_{n} \rightarrow c$ as $n \rightarrow \infty$ for some $0<c<\infty$. By Jensen's inequality,

$$
\sum_{i=1}^{n} \exp \left(-p_{n i} b_{n}\right) \geq \sum_{i=1}^{n} \exp \left(-\frac{1}{n} b_{n}\right)=n \exp \left(-\frac{b_{n}}{n}\right) .
$$

Therefore, $b_{n} \geq n \log n$, and this will be used in Lemma 2.2, below. The only restriction placed upon the sequence $\left\{X^{n}\right\}$ is (2.1). Discussion of a natural construction of suitable sequences $\left\{X^{n}\right\}$ is deferred to Section 3 .

The proof of Theorem 2.1 relies upon two preliminary lemmas which are motivated and proved in the following discussion.

Since, for $t \geq 1, Y_{n} \leq t$ if and only $W_{n}(t)=0$, it suffices to show that, for all $y \in \mathbb{R}$,

$$
W_{n}\left(\left[b_{n}+y k_{n}\right]\right) \stackrel{\mathrm{D}}{\rightarrow} \operatorname{Po}(g(y)), \quad y \in \mathbb{R} .
$$

The first step in proving (2.2) is to show that, for any $t \in \mathbb{N},\left\{\chi_{i}^{n}(t)\right\}$ are negatively related [1, p. 24]. For $n, t \geq 1$ and $1 \leq j \leq n$, let $\left\{\theta_{i, j}^{n}(t) ; i=1,2, \ldots, n\right\}$ be random variables satisfying

$$
\mathcal{L}\left(\theta_{i, j}^{n}(t) ; i=1,2, \ldots, n\right)=\mathcal{L}\left(\chi_{i}^{n}(t) ; i=1,2, \ldots, n \mid \chi_{j}^{n}(t)=1\right) .
$$

Lemma 2.1. For $n, t \geq 1$, the random variables $\left\{\chi_{i}^{n}(t)\right\}$ are negatively related, i.e. for each $1 \leq j \leq n$, the random variables $\left\{\theta_{i, j}^{n}(t) ; i=1,2, \ldots, n\right\}$ and $\left\{\chi_{i}^{n}(t) ; i=1,2, \ldots, n\right\}$ can be defined on a common probability space $(\Omega, \mathcal{F}, \mathrm{P})$ such that, for all $i \neq j, \chi_{i}^{n}(t)(\omega) \geq$ $\theta_{i, j}^{n}(t)(\omega)$ for all $\omega \in \Omega$.

Proof. The lemma is proved by a simple coupling argument.

Fix $n, t \geq 1$ and $j=1,2, \ldots, n$. Draw $X_{1}^{n}, X_{2}^{n}, \ldots, X_{t}^{n}$ from $X^{n}$. For $k=1,2, \ldots, t$, let $\tilde{X}_{k}^{n}(t) \stackrel{\mathrm{D}}{=} X_{k}^{n} \mid \chi_{j}^{n}(t)=1$, where $\stackrel{\text { D }}{=}$, denotes equality in distribution. For $k=1,2, \ldots, t$, if $X_{k}^{n} \neq j$, set $\tilde{X}_{k}^{n}(t)=X_{k}^{n}$. If $X_{k}^{n}=j$, set $\tilde{X}_{k}^{n}(t)=\hat{X}_{k}^{n}$, where

$$
\mathrm{P}\left(\hat{X}_{k}^{n}=i\right)= \begin{cases}\frac{p_{n i}}{1-p_{n j}}, & i \neq j \\ 0, & \text { otherwise. }\end{cases}
$$

Thus, $\tilde{X}_{1}^{n}(t), \tilde{X}_{2}^{n}(t), \ldots, \tilde{X}_{t}^{n}(t)$ have the correct distribution and, by construction, $\chi_{i}^{n}(t) \geq$ $\theta_{i, j}^{n}(t)$ for $i \neq j$.

Note that

$$
\mathrm{E}\left[W_{n}\left(\left[b_{n}+y k_{n}\right]\right)\right]=\sum_{i=1}^{n}\left(1-p_{n i}\right)^{\left[b_{n}+y k_{n}\right]} \rightarrow g(y) \quad \text { as } n \rightarrow \infty .
$$

Therefore, by Lemma 2.1 and [1, Corollary 2.C.2], (2.2) holds if

$$
\operatorname{var}\left(W_{n}\left(\left[b_{n}+y k_{n}\right]\right)\right) \rightarrow g(y) \quad \text { as } n \rightarrow \infty .
$$


Now $\operatorname{var}\left(W_{n}\left(\left[b_{n}+y k_{n}\right]\right)\right)$ is equal to

$$
\sum_{i=1}^{n} \operatorname{var}\left(\chi_{i}^{n}\left(\left[b_{n}+y k_{n}\right]\right)\right)+\sum_{i=1}^{n} \sum_{j \neq i} \operatorname{cov}\left(\chi_{i}^{n}\left(\left[b_{n}+y k_{n}\right]\right), \chi_{j}^{n}\left(\left[b_{n}+y k_{n}\right]\right)\right) .
$$

Equation (2.1) ensures that

$$
\sum_{i=1}^{n} \exp \left(-p_{n i}\left[b_{n}+y k_{n}\right]\right)^{2} \rightarrow 0 \quad \text { as } n \rightarrow \infty .
$$

Therefore, by (2.1), the first term in (2.4) converges to $g(y)$ as $n \rightarrow \infty$. Thus, (2.3) holds if the latter term in (2.4) converges to 0 as $n \rightarrow \infty$.

\section{Lemma 2.2.}

$$
\sum_{i=1}^{n} \sum_{j \neq i}\left|\operatorname{cov}\left(\chi_{i}^{n}\left(\left[b_{n}+y k_{n}\right]\right), \chi_{j}^{n}\left(\left[b_{n}+y k_{n}\right]\right)\right)\right| \rightarrow 0 \text { as } n \rightarrow \infty .
$$

Proof. For any $i \neq j$,

$$
\begin{aligned}
\mid \operatorname{cov} & \left(\chi_{i}^{n}\left(\left[b_{n}+y k_{n}\right]\right), \chi_{j}^{n}\left(\left[b_{n}+y k_{n}\right]\right)\right) \mid \\
& =\left|\left(1-p_{n i}-p_{n j}\right)^{\left[b_{n}+y k_{n}\right]}-\left(1-p_{n i}\right)^{\left[b_{n}+y k_{n}\right]}\left(1-p_{n j}\right)^{\left[b_{n}+y k_{n}\right]}\right| \\
& =\left(1-p_{n i}\right)^{\left[b_{n}+y k_{n}\right]}\left(1-p_{n j}\right)^{\left[b_{n}+y k_{n}\right]}\left|\left(1-\frac{p_{n i} p_{n j}}{\left(1-p_{n i}\right)\left(1-p_{n j}\right)}\right)^{\left[b_{n}+y k_{n}\right]}-1\right| \\
& \leq\left(1-p_{n i}\right)^{[b n \log n+y n]}\left(1-p_{n j}\right)^{\left[b_{n}+y k_{n}\right]} \frac{\left[b_{n}+y k_{n}\right] p_{n i} p_{n j}}{\left(1-p_{n i}\right)\left(1-p_{n j}\right)},
\end{aligned}
$$

with the inequality coming from $\left|1-(1-y)^{m}\right| \leq m y$ for $0 \leq y \leq 1$ and $m \in \mathbb{N}$.

Therefore,

$$
\begin{aligned}
& \sum_{i=1}^{n} \sum_{j \neq i}\left|\operatorname{cov}\left(\chi_{i}^{n}\left(\left[b_{n}+y k_{n}\right]\right), \chi_{j}^{n}\left(\left[b_{n}+y k_{n}\right]\right)\right)\right| \\
& \quad \leq\left(\sqrt{\left[b_{n}+y k_{n}\right]} \sum_{i=1}^{n} \frac{p_{n i}}{1-p_{n i}}\left(1-p_{n i}\right)^{\left[b_{n}+y k_{n}\right]}\right)^{2} .
\end{aligned}
$$

Let $\mathcal{A}_{n}=\left\{i ; p_{n i} \leq b_{n}^{-3 / 4}\right\}$. Then

$$
\begin{aligned}
\sqrt{\left[b_{n}+y k_{n}\right]} \sum_{i=1}^{n} \frac{p_{n i}}{1-p_{n i}}\left(1-p_{n i}\right)^{\left[b_{n}+y k_{n}\right]} \\
=\sqrt{\left[b_{n}+y k_{n}\right]} \sum_{i \in \mathcal{A}_{n}} \frac{p_{n i}}{1-p_{n i}}\left(1-p_{n i}\right)^{\left[b_{n}+y k_{n}\right]} \\
\quad+\sqrt{\left[b_{n}+y k_{n}\right]} \sum_{i \in \mathcal{A}_{n}^{\mathrm{c}}} \frac{p_{n i}}{1-p_{n i}}\left(1-p_{n i}\right)^{\left[b_{n}+y k_{n}\right]} \\
\leq \frac{b_{n}^{-3 / 4} \sqrt{\left[b_{n}+y k_{n}\right]}}{1-b_{n}^{-3 / 4}} \sum_{i=1}^{n}\left(1-p_{n i}\right)^{\left[b_{n}+y k_{n}\right]}+\sqrt{\left[b_{n}+y k_{n}\right]} \sum_{i \in \mathcal{A}_{n}^{\mathrm{c}}}\left(1-b_{n}^{-3 / 4}\right)^{\left[b_{n}+y k_{n}\right]-1} \\
\rightarrow 0 \text { as } n \rightarrow \infty,
\end{aligned}
$$


since $\sum_{i=1}^{n}\left(1-p_{n i}\right)^{\left[b_{n}+y k_{n}\right]} \rightarrow g(y)$ and $b_{n} \geq n \log n$ as $n \rightarrow \infty$. Therefore, the right-hand side of (2.5) converges to 0 as $n \rightarrow \infty$ and the lemma is proved.

Proof of Theorem 2.1. For any $y \in \mathbb{R}, \tilde{Y}_{n} \leq y$ if and only if $W_{n}\left(\left[b_{n}+y k_{n}\right]\right)=0$. Therefore, by (2.2), for $y \in \mathbb{R}$,

$$
\begin{aligned}
\mathrm{P}\left(\tilde{Y}_{n} \leq y\right) & =\mathrm{P}\left(W_{n}\left(\left[b_{n}+y k_{n}\right]\right)=0\right) \\
& \rightarrow \mathrm{e}^{-g(y)} \\
& =\mathrm{P}(Y \leq y) \quad \text { as } n \rightarrow \infty,
\end{aligned}
$$

and the theorem is proved.

The proof of Theorem 2.1 presents a straightforward bound for $\left|\mathrm{P}\left(\tilde{Y}_{n} \leq y\right)-\mathrm{P}(Y \leq y)\right|$, $y \in \mathbb{R}$. For $t \geq 0$, let $Z(t) \sim \operatorname{Po}(t)$ and, for $y \in \mathbb{R}$, let $g_{n}(y)=\mathrm{E}\left[W_{n}\left(\left[b_{n}+y k_{n}\right]\right)\right]$. By the triangle inequality and [1, Corollary 2.C.2],

$$
\begin{aligned}
\mid \mathrm{P}\left(\tilde{Y}_{n}\right. & \leq y)-\mathrm{P}(Y \leq y) \mid \\
& =\left|\mathrm{P}\left(W_{n}\left(\left[b_{n}+y k_{n}\right]\right)=0\right)-\mathrm{P}(Z(g(y))=0)\right| \\
& \leq\left|\mathrm{P}\left(W_{n}\left(\left[b_{n}+y k_{n}\right]\right)=0\right)-\mathrm{P}\left(Z\left(g_{n}(y)\right)=0\right)\right|+\left|\mathrm{P}\left(Z\left(g_{n}(y)\right)=0\right)-\mathrm{P}(Z(g(y))=0)\right| \\
& \leq\left(1-\exp \left(-g_{n}(y)\right)\right)\left(1-\frac{\operatorname{var}\left(W_{n}\left(\left[b_{n}+y k_{n}\right]\right)\right)}{g_{n}(y)}\right)+\left|\exp \left(-g_{n}(y)\right)-\mathrm{e}^{-g(y)}\right| .
\end{aligned}
$$

We now turn our attention to the situation where the natural scaling $\left\{b_{n}\right\}$ is such that $\min _{1 \leq i \leq n} p_{n i} b_{n} \rightarrow c$ as $n \rightarrow \infty$ for some $0<c<\infty$.

Theorem 2.2. Suppose that there exist sequences $\left\{b_{n}\right\}$ such that, for $y \in \mathbb{R}^{+}$,

$$
\sum_{i=1}^{n} \exp \left(-p_{n i} y b_{n}\right) \rightarrow g(y) \quad \text { as } n \rightarrow \infty
$$

for a nonincreasing function $g(\cdot)$ with $g(y) \rightarrow \infty$ as $y \rightarrow 0$ and $g(y) \rightarrow 0$ as $y \rightarrow \infty$.

Suppose that there exists a function $h(\cdot)$ such that, for all $y \in \mathbb{R}^{+}$,

$$
\prod_{i=1}^{n}\left(1-\exp \left(-p_{n i} y b_{n}\right)\right) \rightarrow h(y) \text { as } n \rightarrow \infty \text {. }
$$

Then (2.6) ensures that $h(y) \rightarrow 0$ as $y \rightarrow 0$ and $h(y) \rightarrow 1$ as $y \rightarrow \infty$, and if $\hat{Y}_{n}=Y_{n} / b_{n}$,

$$
\hat{Y}_{n} \stackrel{\mathrm{D}}{\rightarrow} Y \quad \text { as } n \rightarrow \infty,
$$

where $Y$ has cumulative distribution function

$$
\mathrm{P}(Y \leq y)=h(y), \quad y \in \mathbb{R}^{+} .
$$

Proof. The proof has a number of similarities and differences to the proof of Theorem 2.1. We shall again exploit the fact that $Y_{n} \leq t$ if and only $W_{n}(t)=0$.

Let $\eta_{*}^{n}$ be a homogeneous Poisson point process with rate 1 , and let $T_{n}(t)$ denote the time of the $\left[t b_{n}\right]$ th point on $\eta_{*}^{n}$. Let $V_{1}^{n}, V_{2}^{n}, \ldots$ be independent and identically distributed according to $X^{n}$. Let $\eta_{1}^{n}, \eta_{2}^{n}, \ldots, \eta_{n}^{n}$ be independent homogeneous Poisson point processes with rates 
$p_{n 1}, p_{n 2}, \ldots, p_{n n}$, respectively, constructed from $\eta_{*}^{n}$ and $V_{1}^{n}, V_{2}^{n}, \ldots$ as follows. For $k=$ $1,2, \ldots$, let $s_{k}^{n}$ denote the time of the $k$ th point on $\eta_{*}^{n}$. Then there is a point on $\eta_{j}^{n}$ at time $s_{k}^{n}$ if $V_{k}^{n}=j$. Furthermore, $\chi_{1}^{n}(t), \chi_{2}^{n}(t), \ldots, \chi_{n}^{n}(t)$ and, hence, $W_{n}(t)$ can be constructed using $V_{1}^{n}, V_{2}^{n}, \ldots, V_{t}^{n}$.

Let $\psi_{i}^{n}(t)=1$ if there is no point on $\eta_{i}^{n}[0, t]$, and note that the $\left\{\psi_{i}^{n}(t)\right\} \mathrm{s}$ are independent. For $t \geq 0$, let $\tilde{W}_{n}(t)=\sum_{i=1}^{n} \psi_{i}^{n}(t)$. Then $W_{n}\left(\left[y b_{n}\right]\right)=\tilde{W}_{n}\left(T_{n}\left(\left[y b_{n}\right]\right)\right)$. Since $\tilde{W}_{n}(\cdot)$ is nondecreasing, if $\left[y b_{n}\right]-\left(\left[y b_{n}\right]\right)^{3 / 4} \leq T_{n}\left(\left[y b_{n}\right]\right) \leq\left[y b_{n}\right]+\left(\left[y b_{n}\right]\right)^{3 / 4}$ then

$$
\tilde{W}_{n}\left(\left[y b_{n}\right]+\left(\left[y b_{n}\right]\right)^{3 / 4}\right) \leq W_{n}\left(\left[y b_{n}\right]\right) \leq \tilde{W}_{n}\left(\left[y b_{n}\right]-\left(\left[y b_{n}\right]\right)^{3 / 4}\right) .
$$

Since $\left(1 /\left(y b_{n}\right)^{3 / 4}\right)\left(T_{n}\left(\left[y b_{n}\right]\right)-\left[y b_{n}\right]\right) \stackrel{\mathrm{P}}{\rightarrow} 0$ as $n \rightarrow \infty$ (where $\stackrel{\text { P }}{\rightarrow}$ ' denotes convergence in probability), it follows from $(2.7)$ that $\mathrm{P}\left(W_{n}\left(\left[y b_{n}\right]\right)=0\right) \rightarrow h(y)$ if

$$
\mathrm{P}\left(\tilde{W}_{n}\left(\left[y b_{n}\right] \pm\left(\left[y b_{n}\right]\right)^{3 / 4}\right)=0\right) \rightarrow h(y) \quad \text { as } n \rightarrow \infty .
$$

By independence, for all $y \in \mathbb{R}$,

$$
\begin{aligned}
\mathrm{P}\left(\tilde{W}_{n}\left(\left[y b_{n}\right] \pm\left(\left[y b_{n}\right]\right)^{3 / 4}\right)=0\right) & =\prod_{i=1}^{n}\left(1-\left(1-p_{n i}\right)^{\left[y b_{n}\right] \pm\left(\left[y b_{n}\right]\right)^{3 / 4}}\right) \\
& \rightarrow h(y) \quad \text { as } n \rightarrow \infty .
\end{aligned}
$$

The main benefit of Theorem 2.1 over Theorem 2.2 is that $g(y)$ is usually much easier to calculate than $h(y)$.

\section{Examples}

A natural construction of $\left\{X^{n}\right\}$ is to take a (continuous) distribution $X$ with probability density function $f(\cdot)$ on $[0,1]$ and, for $n=1,2, \ldots$ and $i=1,2, \ldots, n$, set

$$
p_{n i}=\int_{(i-1) / n}^{i / n} f(x) \mathrm{d} x .
$$

A number of results can be proved concerning various choices of $X$ with Lemma 3.1 illustrating the point using a class of distributions with $f(\cdot)$ being continuous.

Lemma 3.1. Let $0 \leq \sigma \leq 1$ be such that, for all $0 \leq x \leq 1$ and $x \neq \sigma, 0<f(\sigma)<f(x)$. For $p=1,2$, let

$$
u_{p}=\lim _{\varepsilon \rightarrow 0+} \frac{f(\sigma+\varepsilon)-f(\sigma)}{\varepsilon^{p}}, \quad l_{p}=\lim _{\varepsilon \rightarrow 0-} \frac{f(\sigma+\varepsilon)-f(\sigma)}{|\varepsilon|^{p}} .
$$

(i) Suppose that $\mathbf{1}_{\{\sigma>0\}} l_{1}+\mathbf{1}_{\{\sigma<1\}} u_{1}>0$. Then $b_{n}=(n / f(\sigma))(\log n-\log (\log n))$ and $k_{n}=n$ with

$$
g(y)=f(\sigma)\left(\frac{\mathbf{1}_{\{\sigma>0\}}}{l_{1}}+\frac{\mathbf{1}_{\{\sigma<1\}}}{u_{1}}\right) \mathrm{e}^{-f(\sigma) y} .
$$

(ii) Suppose that $\mathbf{1}_{\{\sigma>0\}} l_{1}+\mathbf{1}_{\{\sigma<1\}} u_{1}=0$ and $\mathbf{1}_{\{\sigma>0\}} l_{2}+\mathbf{1}_{\{\sigma<1\}} u_{2}>0$. Then $b_{n}=$ $(n / f(\sigma))\left(\log n-\frac{1}{2} \log (\log n)\right)$ and $k_{n}=n$ with

$$
g(y)=\sqrt{\frac{\pi f(\sigma)}{2}}\left(\sqrt{\frac{\mathbf{1}_{\{\sigma>0\}}}{l_{2}}}+\sqrt{\frac{\mathbf{1}_{\{\sigma<1\}}}{u_{2}}}\right) \mathrm{e}^{-f(\sigma) y} .
$$


Proof. We outline the proof of (i), with (ii) being proved similarly.

Let $b_{n}=(n / f(\sigma))(\log n-\log (\log n))$ and $k_{n}=n$. Note that

$$
\begin{aligned}
\sum_{i=1}^{n} \exp \left(-p_{n i}\left(b_{n}+y k_{n}\right)\right) & \approx \sum_{i=1}^{n} \exp \left(-\left(b_{n}+y k_{n}\right) \frac{1}{n} f\left(\frac{i-1 / 2}{n}\right)\right) \\
& =n \sum_{i=1}^{n} \frac{1}{n} \exp \left(-\left(\frac{b_{n}}{n}+y\right) f\left(\frac{i-1 / 2}{n}\right)\right) \\
& \approx n \int_{0}^{1} \exp \left(-\left(\frac{b_{n}}{n}+y\right) f(x)\right) \mathrm{d} x
\end{aligned}
$$

Therefore, it is straightforward to show that

$$
g(y)=\lim _{n \rightarrow \infty} n \int_{0}^{1} \exp \left(-\left(\frac{1}{f(\sigma)}(\log n-\log (\log n))+y\right) f(x)\right) \mathrm{d} x .
$$

Linearizing $f(x)$ about $\sigma$ and considering the left- and right-hand limits separately yields the result.

Examples of probability density functions on $[0,1]$ satisfying Lemma 3.1 include $f(x)=$ $\frac{2}{3}(1+x), f(x)=\frac{6}{5}(1-x(1-x))$, and $f(x)=\frac{12}{7} \max (1-x, x / 2)$.

Suppose instead that $X$ is piecewise constant with, for $1 \leq j \leq k$,

$$
f(x)=\lambda_{j}, \quad \pi_{j-1}<x \leq \pi_{j},
$$

where $\lambda_{1}, \lambda_{2}, \ldots, \lambda_{k}>0$ and $0=\pi_{0}<\pi_{1}<\cdots<\pi_{k}=1$. Without loss of generality, assume that $\lambda_{1}<\lambda_{2}<\cdots<\lambda_{k}$. Then $b_{n}=\left(1 / \lambda_{1}\right) n \log n, k_{n}=n$, and $g(y)=$ $\pi_{1} \exp \left(-\lambda_{1} y\right)$.

In the above examples, $k_{n} / b_{n} \rightarrow 0$ and Theorem 2.1 holds. In all cases, the limiting distribution $Y$ is a Gumbel distribution with $b_{n} / n \log n \rightarrow 1 / \min _{0 \leq x \leq 1} f(x)$ as $n \rightarrow \infty$.

An example of where Theorem 2.2 is necessary is $f(x)=2 x(0 \leq x \leq 1)$, giving $p_{n i}=$ $(2 i-1) / n^{2}(i=1,2, \ldots, n)$. Then, for $y \in \mathbb{R}^{+}$,

$$
\sum_{i=1}^{n} \exp \left(-p_{n i} y n^{2}\right)=\sum_{i=1}^{n} \exp (-(2 i-1) y) \rightarrow g(y)=\frac{\mathrm{e}^{y}}{\mathrm{e}^{2 y}-1} \quad \text { as } n \rightarrow \infty,
$$

and Theorem 2.2 holds with $b_{n}=n^{2}$ and $h(y)=\lim _{n \rightarrow \infty} \prod_{i=1}^{n}(1-\exp (-(2 i-1) y))$.

\section{Extensions}

The methodology outlined in Section 2 can be extended to find the total number of coupons, $Y_{n}^{K}$, which need to be collected in order to have (at least) $K$ coupons of each type. In this case, simply let $\chi_{i}^{n}(t)=1$ if at most $K-1$ coupons of type $i$ have been collected in the first $t$ draws from the population and let $\chi_{i}^{n}(t)=0$ otherwise. Then set $W_{n}^{K}(t)=\sum_{i=1}^{n} \chi_{i}^{n}(t)$, and note that $Y_{n}^{K} \leq t$ if and only if $W_{n}^{K}(t)=0$. It is straightforward to adapt Lemmas 2.1 and 2.2 to this case and, consequently, Theorem 2.1 holds with (2.1) replaced by

$$
\frac{b_{n}^{K-1}}{(K-1) !} \sum_{i=1}^{n} p_{n i}^{K-1} \exp \left(-p_{n i}\left\{b_{n}+y k_{n}\right\}\right) \rightarrow g(y) \quad \text { as } n \rightarrow \infty .
$$


Since $k_{n} / b_{n} \rightarrow 0$ implies that $\min _{1 \leq i \leq n} b_{n} p_{n i} \rightarrow \infty$ as $n \rightarrow \infty,(4.1)$ holds if and only if

$$
\mathrm{E}\left[W_{n}^{K}\left(\left[b_{n}+y k_{n}\right]\right)\right] \rightarrow g(y) \quad \text { as } n \rightarrow \infty .
$$

Theorem 2.2 can also be adapted to the $K$-coupon collector problem.

At the other end of the spectrum, the Poisson arguments above can be applied to the generalised birthday problem. That is, for $K \geq 2$, let $U_{n}^{K}$ denote the total number of draws from the population that are required to obtain $K$ coupons of any (unspecified) type. Let $\tilde{\chi}_{i}^{n}(t)=1$ if at least $K$ coupons of type $i$ have been collected in the first $t$ draws from the population and let $\tilde{\chi}_{i}^{n}(t)=0$ otherwise. Then, if $\tilde{W}_{n}^{K}(t)=\sum_{i=1}^{n} \tilde{\chi}_{i}^{n}(t), U_{n}^{K}>t$ if and only if $\tilde{W}_{n}^{K}(t)=0$. Along the lines of Lemma 2.1, it can be shown that the $\left\{\tilde{\chi}_{i}^{n}(t)\right\}$ are negatively related and straightforward bounds for the covariance terms can be obtained. We then have the following theorem.

Theorem 4.1. For fixed $K \geq 2$, suppose that there exists a sequence $\left\{l_{n}\right\}$ such that

$$
l_{n}^{K} \sum_{i=1}^{n} p_{n i}^{K} \rightarrow 1
$$

and $\max _{1 \leq i \leq n} l_{n} p_{n i} \rightarrow 0$ as $n \rightarrow \infty$. Then

$$
\frac{U_{n}^{K}}{l_{n}} \stackrel{\mathrm{D}}{\rightarrow} U^{K} \quad \text { as } n \rightarrow \infty,
$$

where $U^{K}$ has cumulative distribution function

$$
\mathrm{P}\left(U^{K} \leq u\right)=1-\exp \left(-u^{K}\right), \quad u \in \mathbb{R}^{+} .
$$

Proof. The conditions imposed on $\left\{l_{n}\right\}$ are sufficient for $W_{n}^{K}\left(\left[u l_{n}\right]\right) \stackrel{\mathrm{D}}{\rightarrow} \operatorname{Po}\left(u^{K}\right)$, from which the theorem follows immediately.

The limiting distribution $U^{K}$ obtained in Theorem 4.1 is identical to that obtained in [4, Theorem 5.2] for the random birthday problem. For the case in which $K=2$, Theorem 4.1 follows immediately from [2, Example 2], since given (4.2), $\max _{1 \leq i \leq n} l_{n} p_{n i} \rightarrow 0$ if and only if $l_{n}^{3} \sum_{i=1}^{n} p_{n i}^{3} \rightarrow 0$ as $n \rightarrow \infty$.

Finally, it is worth noting that, for the establishing of Poisson limits for $W_{n}^{K}\left(\left[b_{n}+y k_{n}\right]\right)$ and $\tilde{W}_{n}^{K}\left(\left[u l_{n}\right]\right)$, it is crucial that

$$
\max _{1 \leq i \leq n} \mathrm{E}\left[\chi_{i}^{n}\left(\left[b_{n}+y k_{n}\right]\right)\right] \rightarrow 0 \quad \text { and } \max _{1 \leq i \leq n} \mathrm{E}\left[\tilde{\chi}_{i}^{n}\left(\left[u l_{n}\right]\right)\right] \rightarrow 0 \quad \text { as } n \rightarrow \infty,
$$

respectively. That is, for the $K$-coupon collector problem, we require that $\min _{1 \leq i \leq n} b_{n} p_{n i} \rightarrow \infty$ as $n \rightarrow \infty$ (none of the probabilities are too small) and, for the $K$-birthday problem, we require that $\max _{1 \leq i \leq n} l_{n} p_{n i} \rightarrow 0$ as $n \rightarrow \infty$ (none of the probabilities are too large).

\section{Acknowledgement}

I would like to thank John Moriarty for helpful discussions, in particular suggesting the construction of $\left\{X^{n}\right\}$ used in Section 3. 


\section{References}

[1] Barbour, A. D., Holst, L. And Janson, S. (1992). Poisson Approximation. Oxford University Press.

[2] Blom, G. ANd Holst, L. (1989). Some properties of similar pairs. Adv. Appl. Prob. 21, 941-944.

[3] Feller, W. (1957). An Introduction to Probability Theory and Its Applications. John Wiley, New York.

[4] Holst, L. (2001). Extreme value distributions for random coupon collector and birthday problems. Extremes 4, 129-145.

[5] Papanicolaou, V. G., Kokolakis, G. E. and Boneh, S. (1998). Asymptotics for the random coupon collector problem. J. Comput. Appl. Math. 93, 95-105. 